\title{
Relationship between compression pressure, mechanical strength and release properties of tablets
}

\author{
Olutayo Ademola Adeleye ${ }^{A-F}$
}

Olabisi Onabanjo University, Ago-Iwoye, Nigeria

A - research concept and design; $B$ - collection and/or assembly of data; $C$ - data analysis and interpretation;

$D$ - writing the article; $E$ - critical revision of the article; $F$ - final approval of the article

Polymers in Medicine, ISSN 0370-0747 (print), ISSN 2451-2699 (online)

Polim Med. 2019;49(1):27-33

\section{Address for correspondence \\ Olutayo Ademola Adeleye}

E-mail: olutayoadeleye@yahoo.com

\section{Funding sources}

None declared

Conflict of interest

None declared

Received on March 15, 2019

Reviewed on May 12, 2019

Accepted on August 22, 2019

Published online on November 18, 2019

Cite as

Adeleye $\mathrm{OA}$. Relationship between compression pressure, mechanical strength and release properties of tablets.

Polim Med. 2019;49(1):27-33. doi:10.17219/pim/111888

DOI

10.17219/pim/111888

\section{Copyright}

○) 2019 by Wroclaw Medical University

This is an article distributed under the terms of the

Creative Commons Attribution 3.0 Unported (CC BY 3.0)

(https://creativecommons.org/licenses/by/3.0/)

\begin{abstract}
Tablets are a complex drug delivery system consisting of the active pharmaceutical ingredients and excipients. Tablet production involves a series of unit operations in which drugs and excipients are subjected to mechanical stresses, such as compression pressure, thus imposing changes in the properties of these materials. Variations in the compression pressure and other processing parameters may affect the mechanical strength and release properties of the final tablet. It is generally expected that an increase in compression pressure should lead to an increase in mechanical strength and a decrease in release properties of tablets, but this may not be true in some practical situation, since tablet production is the result of complex interaction between many factors involving the drug, excipient, the formulation, and processing variables. The degree and extent of interaction of these variables are not absolutely dependent on one factor. The aim of this review is to study the interaction between compression pressure, mechanical strength and release properties of immediate and controlled release tablets. The effect of compression pressure on tablets is complemented by such factors as the material properties of the drug and excipient, the formulation and processing factors, which in turn affects mechanical strength and release properties.
\end{abstract}

Key words: mechanical properties, dissolution, compression pressure, release properties, crushing strength 


\section{Introduction}

Tablets are a complex drug delivery system consisting of the drug substance and adjuvants known as excipients, which aid the formulation of the desired drug product. Polymers as excipients in tablet production play an important role in determining the properties of a finished tablet. They have been extensively used in the design of various drug delivery systems as binders, disintegrants, drug release modifiers, directly compressible excipient, film coating, emulsifiers, suspending agents, thickeners, gelling agents, nanoparticles, etc.

Tablet production involves a series of unit operations usually consisting of mixing, blending and compaction. Other operations, such as granulation, coating, etc., may also be used depending on the type and desired properties of the finished product. During each unit operation process, drugs and excipients are subjected to mechanical stresses, which impose changes in the properties of these materials. This process is complex, because the knowledge of the properties of these starting material is often not complete, and their relationship to formulation factors and processing parameters is frequently not understood well; hence the essence of preformulation characterization to predict quality of the end product. ${ }^{1}$ Also, producing a tablet of desirable quality requires understanding of the physics of compression. ${ }^{2}$ Slight variations in formulation factors, processing parameters and material properties may affect the quality of the final tablet. The active pharmaceutical ingredients (APIs) and excipients, especially the binders, ${ }^{3}$ disintegrants ${ }^{4}$ and lubricants ${ }^{5}$ used in tablet production, determine tablet properties. Compression pressure $^{6}$ and method of production ${ }^{3,7}$ also contribute to the final tablet properties.

In general, tablet production involves the application of pressure (i.e., compression pressure) to a powder bed, compressing it into a cohesive compact through a process known as compaction. ${ }^{8}$ Compaction is a simultaneous process of compression and consolidation of a particulate solid-gas system as a result of applied force. ${ }^{9,10}$ While compression involves a reduction in bulk volume as a result of reduced gaseous phase with an increase in pressure within a powder bed, thereby resulting in closer packing of the powder particles, consolidation occurs simultaneously as force is increased to a point where further rearrangement becomes difficult and particle deformation occurs, leading to an increase in mechanical strength as a result of particle-particle interactions which result in bond creation. Consolidation is mainly responsible for increasing the mechanical strength of a powder bed under a rising compressive force. ${ }^{11}$

The aim of this review is to study the interaction between compression pressure (independent variable), mechanical strength and release properties (dependent variables) of immediate and controlled release tablets produced through wet granulation or direct compression techniques.

\section{Compression pressure}

Compression pressure is an external axial force which occurs when a physical force is pressed inward on the solid surface of an object, deforming it either permanently or temporarily, depending on the material and the magnitude of the force. The extent, degree and the direction of the deformation depend on such factors as the properties of the APIs and excipients, the type and concentration of ingredients in the formulation, particle size, particle shape, molecular weight, solubility, formulation processes, wetting properties, polymer properties, type of binding force that exist during consolidation, etc.

These are the critical quality attributes that determine the optimized force needed to make a tablet of good quality. This force is determined mostly during preformulation studies. The United States Pharmacopeia and the European Pharmacopeia did not specify a limit of compression pressure required to produce a cohesive compact, and therefore its determination is often empirical depending on the critical quality attributes of the material to be compressed. Compression pressure is an important control parameter, because it influences almost every tablet property, such as density, porosity, surface area, hardness, friability, disintegration, dissolution, etc. ${ }^{12}$

Density increases with the increase in compression force, while porosity decreases. Hardness increases with the increase in compression force, while friability decreases. ${ }^{13}$ In many cases, an exponential relationship exists between disintegration time and compression pressure. The disintegration time increases with the increase in pressure. There is no clear-cut trend with dissolution. This may depend on the type of tablet, such as immediate release (disintegrating tablets) and controlled release (non-disintegrating tablets).

Tablet hardness, crushing strength or tensile strength is used in many cases as a surrogate measure for compression pressure during tablet production, since tableting machines are unable to determine and measure the compression force that is desired. ${ }^{14}$

The effect of compression pressure on tablets is complemented by the material properties and the formulation factors, which in turn may affect mechanical strength and release properties.

\section{Mechanical properties of tablets}

The ability of a tablet to withstand mechanical handling and transport is a measure of tablet mechanical strength, which is an important and widely used parameter to control the production process. The major tests available for the assessment of the mechanical properties of tablets are the hardness $(\mathrm{N}$ or Kgf $)$, tensile strength $\left(\mathrm{kg} / \mathrm{cm}^{2}\right)$ and friability (\% loss). Mechanical strength assessment generally begins with mechanical testing of particles of drugs and excipients with compression parameters derived from 
Heckel $^{15}$ and Kawakita and Lüdde, ${ }^{16}$ which are the most commonly used compaction equations. These compaction equations are based on the relationship between applied load and volume reduction of materials in the die and none has been found to be fully satisfactory.

Some of the factors generally affecting tablet mechanical strength are:

Particle size - a decrease in particle size will result in increased tablet mechanical strength; powders of very fine particles are cohesive even in their uncompressed form.

Moisture content - a small proportion of moisture is essential for the production of a cohesive tablet. In some cases, wet granulation process with hydrophilic polymer was reported to yield tablet with stronger mechanical strength, ${ }^{3,7}$ and, thus, there should be optimum moisture content for the production of a good quality tablet.

Lubricants - they act as a physical barrier which prevents particle-particle interactions affecting inter-particulate bond formation, thereby decreasing tablets mechanical strength.

Compression pressure - during compression, an external force is introduced which causes fragmentation, resulting in the formation of new surfaces with increased surface area; thus, more area and particles are available for bond formation. A linear relationship exists between tablet crushing strength and compression pressure, except at high forces.

\section{Hardness}

Tablet hardness or breaking strength is a measure of tablet strength. It is the force required to break a tablet from which tensile strength is determined. It assesses how strong a tablet has to be to withstand breakage, crumbling or chipping under the conditions of storage, transportation and handling. ${ }^{17}$ It generally depends on the type and concentration of binder in a formulation, ${ }^{18}$ tablet height to diameter ratio ${ }^{12}$ and compression force. ${ }^{6,19}$

\section{Friability}

Tablet friability is a measure of the resistance of tablets to fracture. The test is generally used to assess the ability of tablets to withstand shock and abrasion, which will unavoidably be encountered during manufacturing, packaging, transportation, and handling. ${ }^{20}$

\section{Effect of compression pressure on mechanical strength}

Generally, there is an increase in mechanical strength with increased compression pressure. Sun and $\mathrm{HaO}^{21} \mathrm{ob}-$ served that the tensile strength of caffeine tablets was reasonably and substantially higher than that of methyl gallate produced under the same compression pressure gauge. They also observed a sharp reduction in tablet tensile strength of both tablets at a point with increasing pressure, suggesting that the detrimental effect of elastic recovery on tablet strength supersede the bonding strength gained from increased compression pressure. However, the formation of 1:1 cocrystal of caffeine and methyl gallate exhibited superior compaction properties due to the presence of slip planes in crystal structure, thereby improving tablet strength with increasing pressure. Changes in the tensile strength of caffeine tablets, methyl gallate tablets and the co-crystal combination of caffeine and methyl gallate tablet was due to the material properties of the drugs, since that was the only variable in the study. ${ }^{21}$

A study carried out by Adeleye et al. ${ }^{6}$ established that compression pressure is a strong variable which has a great impact on the hardness of tablets when all other manufacturing processes are kept constant. Statistical analysis revealed a significant increase in tablet hardness with increase in compression pressure. This was due to gas displacement from the powder bed in the die as compression pressure increases, bringing particles in close contact, thereby leading to the formation of a strong bond, which, in turn, increases the mechanical strength of the tablet at high compression pressure. They also observed a decrease in friability with increase in compression pressure. The decrease was attributed to the formation of more solid bonds, which increases hardness, thus making tablets more resistant to fracture and abrasion. ${ }^{17}$

Nanjwade et al. ${ }^{22}$ reported an increase in the breaking force and pellet hardness of cefuroxime axetil along with an increase in compression pressure as a result of the existence of a very firm intrinsic compact binding property between the individual particles of the drug and the excipient, which ultimately resulted in a decrease in in-vitro dissolution.

Shipar et al. $^{23}$ and Vodáčková et al. ${ }^{12}$ concluded that compression pressure has an intense effect on tablet hardness and disintegration time. Tablet hardness and disintegration time increases with increasing compression pressure. According to Ordu et al., ${ }^{24}$ excess amounts of binders and compression pressure may lead to the production of tablets that are too hard, which may affect disintegration taking place within the desired time.

\section{Release properties of tablets}

The significance, magnitude and extent of the effect of variables on the release properties lie mostly in the nature of materials in the formulation rather than in compression pressure. Ordu and Onyemelukwe $\mathrm{e}^{25}$ reported that the nature or form of excipient, especially the binder, had a considerable influence on the release properties of a tablet. The concentration is the most important independent variable, as it had more influence on other variables, such as compression pressure, affecting the release properties of tablets. In some cases, compression pressure does not have a significant effect on the release properties of tablets. 


\section{Tablet disintegration}

The first step towards dissolution is breaking down the tablet into smaller particles or granules in conventional tablets. This process is known as disintegration. It exposes a greater surface area of tablets to the dissolution medium; hence, it plays an importance role in tablet dissolution for the active drug substance to be released into the absorption site. ${ }^{26}$

The disintegration time of tablets usually depends on formulation factors such as the nature, type and amount of drug and the excipients used (this could be referred to as internal variables), as well as on the actual tablet manufacturing conditions such as compression force, speed of compression and environmental conditions of the room where the compression is taking place (this could be referred to as the external variables). Often, disintegration time increases exponentially with an increase in compression pressure. Tablets made with high compression pressure exhibit low porosity with strong inter-particulate bond, thereby increasing the time required for penetration of water into the tablet, thus increasing the disintegration time. In tablets made with low compression pressure, there is large porosity and the bond is weak, thereby decreasing the time required for penetration of water into the tablet, thus decreasing the disintegration time. Mistry et al. ${ }^{27}$ reported that binder concentration has a direct relationship with hardness and disintegration and an inverse relationship with friability. As the amount/concentration of the binder increases, the hardness and disintegration times also increases, while the friability decreases.

\section{Tablet dissolution}

Dissolution is the process by which a solid drug goes into solution in a solvent. Drug dissolution is an important condition for the bioavailability of drugs. The dissolution rate of a drug is the most important factor determining drug absorption; it is the rate-limiting step in many formulations. ${ }^{28}$ Therefore, dissolution time gives an indication of the time needed for the drug to be absorbed and to induce a therapeutic effect. Dissolution is a multistep process involving different interactions between the drug and the dissolution medium. This process involves the release of drug molecules from the tablet surface with the formation of hydrated molecules at the solid-liquid interface, and the mass transport of the drug molecules from the interphase to the bulk solution. ${ }^{29}$

Controlled drug delivery tablet systems are designed for tablets to remain intact in the gastrointestinal tract, where they gradually erode to release their content. If the structure or design is lost, drug release is no longer controlled and the tablet behaves like a conventional immediate release tablet, causing dose dumping. Tablet hardness has no significant influence on the dissolution rate of this system. ${ }^{6}$ It is only important here to guide the formulator to produce tablets that can withstand shock and abrasion during production and handling. Drug release mechanisms are complex processes involving swelling, erosion and diffusion across tablet surfaces, in which hardness and compression force may not have any significant influence. ${ }^{30}$

\section{Effect of compression pressure on release properties}

Several authors stated that compression pressure is a statistically significant factor of tablet hardness, but its effect on drug release was found to be minimal. ${ }^{31}$ Some authors observed that compression does not have any effect on the release properties of drugs, ${ }^{32}$ while some observed that it does. ${ }^{22,33}$

The ideal theoretical relationship between dissolution and compression pressure is dissolution being slowed with an increase in compression pressure; however, the increase in force of compression may not affect the dissolution rate of a drug in that manner. The possible relationships that can occur between dissolution and compression pressure are the following: dissolution being more rapid with an increase in pressure, dissolution being slower with an increase in pressure, dissolution being faster to a maximum with an increase in pressure and later becoming slower with a further increase in compression pressure, and dissolution being slower to a minimum with an increase in pressure and later becoming fast with a further increase in compression pressure. Dissolution mostly depends upon the nature of the drug (solid properties, solubility, etc.) and also upon the nature (compactibility) and the amount of binder present in the formulation. An increase in compression force will result in a slower rate of dissolution of some types of tablets due to factors which include hardness (this increase is a result of high compact arrangement of the particles in the tablet, thus making it difficult for the dissolution medium to penetrate the tablet), surface area (due to the compact arrangement of particles, the surface area of the materials in the tablet is reduced, which means that the exposed surface to the dissolution medium is also reduced, resulting in a reduction in dissolution rate of tablets) and wettability (the wettability of the tablet decreases in the dissolution medium due to the compact arrangement of particles, slowing down the penetrability of the dissolution medium into the tablet core, resulting in a reduction in the dissolution rate of tablets).

Dedhiya et al. $^{34}$ reported that the release properties of lithium carbonate did not significantly change with the increasing compression force. Iranloye and Parrott ${ }^{35}$ also reported that compression pressure had no effect on the dissolution rates at a certain range.

Adeleye et al. ${ }^{6}$ tried to relate compression pressure with the drug release rate, found that drug release is controlled mainly by diffusion and concluded that compression pres- 
sure does not have a significant effect on the release rate and release mechanisms, but rather the polymer and the material properties of the drug. This was in agreement with the report of Santos et al. ${ }^{32}$

Generally, drug release from matrix tablets takes place as a result of hydration, when the matrix comes in contact with dissolution medium, ${ }^{36}$ which leads to the formation of gel in which drug diffuses out. The viscosity of this gel layer and its diffusional path length determine the release rate rather than the compression pressure or tablet hardness. ${ }^{37,38}$ These factors (viscosity and diffusional path length of the gel layer) are the rate-determining step in matrix tablet dissolution.

The effect of compression force on dissolution behavior of a drug may be significant or insignificant. It mainly depends upon the nature of the drug and the excipients included. For example, the effect of compression will be negligible on the dissolution rate of a water-soluble drug. For a water-insoluble drug with low melting point, e.g., erythromycin stearate, compression force has an enormous effect on the dissolution rate of such drug, which could lead to failed disintegration and dissolution tests. Increasing the compression force of such material with low melting point will lead to the formation of a complex due to heat generated during compression, which reduces the dissolution rate. For such material, compression force should be optimized.

Nanjwade et al. ${ }^{22}$ reported a sharp decline in the release of cefuroxime axetil with an increase in compression pressure. The release was directly proportional to compression pressure. Cefuroxime axetil has good compression, strong binding and gelling properties. They concluded that compaction by means of compression pressure of cefuroxime axetil is a major reason for a drastic drop of dissolution.

Marais et al. ${ }^{39}$ reported that an increase in compression force increased the disintegration times of furosemide tablets, but did not have a significant effect on the dissolution of the tablet. They concluded that dissolution is mainly a reflection of the disintegration time, which depends on the concentration of disintegrant in the formulation. It was assumed that his was due to the higher concentration of disintegrant, which improved the rate and extent of liquid uptake and penetration into the tablets, allowing them to break up easily and rapidly, thus exposing the drug particles to the dissolution medium very quickly. Audu-Peter and Ibrahim ${ }^{40}$ observed in their study that some tablet formulations which do not have satisfactory disintegration time could not release up to $50 \%$ of their drug content at the end of a 60-minute observation period, while some were able to release up to $100 \%$, despite not having a satisfactory disintegration time. All formulations with satisfactory disintegration time had good release values of up to $95 \%$ after 60 -minute observation period. The reasons why formulations with unsatisfactory disintegration time had a good release profile was attributed to low binder concentration, high concentration of disintegrant and low compression force, while satisfactory disintegration time with lower release was attributed to high compression force and the proportions of binder and disintegrant in the formulation. Ordu et al., ${ }^{24}$ Ordu and Onyemelukwe, ${ }^{25}$ and Onyishi et al. ${ }^{41}$ also reported the effect of ratio and the amount of binder and disintegrant concentrations on release properties.

Figure 1 depicts the schematic representation of the theoretical concept of the relationship between compression pressure, mechanical strength and release properties of a tablet as conceived by the researcher. This model has 3 different variables divided into 2 categories: independent and dependent variables. The independent variable is the compression pressure while the dependent variables are the mechanical strength (hardness and friability) and release properties (disintegration and dissolution). Each of the dependent variables is related to compression pressure and to each other as reflected in the model.

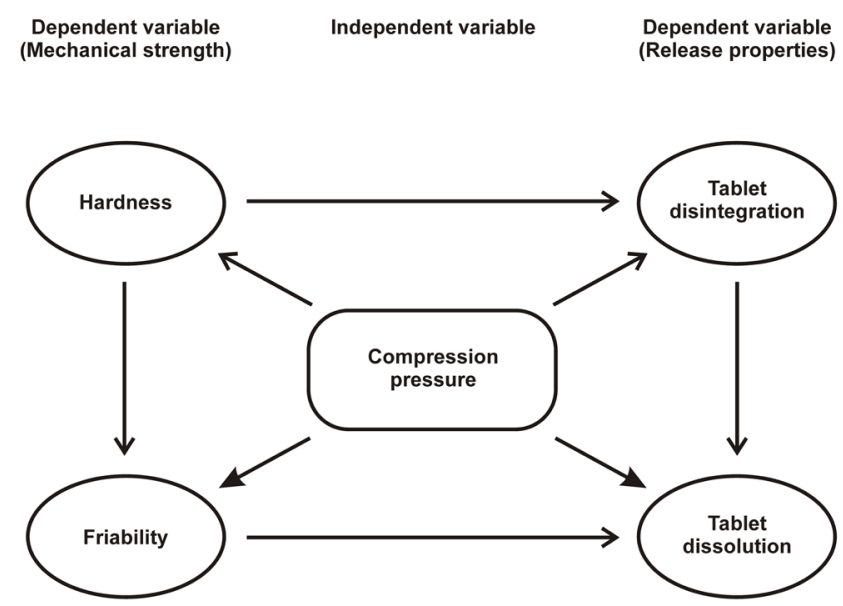

Fig. 1. Schematic representation of the relationship between compression pressure, mechanical strength and release properties of tablet

\section{Discussion}

Several factors affecting tablet quality during its preparation come into play. Such factors can be classified as internal and external variables. The internal factors are the nature, properties and concentration of excipient and drug, while the external factors include compression method (direct compression or wet granulation), compression pressure and compression speed. The magnitude of the influence of these variables is important to the pharmaceutical formulator to guide in the careful selection of excipients, formulation factors and processes to produce tablet of good property.

Properties of various tablet formulations vary because of the existence of different forms of interactions between the drug and the excipients as a result of the operations involved during manufacturing. Properties such as the nature, concentration, particle size, particle shape, mo- 
lecular weight, solubility, etc. are some of the properties that influence the interaction, together with the external factors as enumerated above. The effect of these interactions can be minimized or maximized by modifying these properties to produce tablets of desired properties. The adhesion between powder materials is a result of the area of close contact between particles and the strength of such interactions. The quantity of the interaction is determined by the degree of compression as well as material properties, such as particle size and morphology, while the quality of interaction is determined by the surface properties, such as free surface energy. ${ }^{42}$

The greatest variables, which have overwhelming effects on tablet properties, generally occurred between type, nature and concentration of the binder, mostly due to the fact that the nature of the binder determines its plastoelastic properties and the magnitude of plastic deformation that will take place under compression forces. ${ }^{43}$

In tablet production, Audu-Peter and Ibrahim ${ }^{40}$ reported that there should be a balance between formulation variables for the production of a tablet with good property. Some situations demand an appropriate ratio of binder and compression pressure; otherwise, excessive binder content may produce very hard granules that may not be properly compressed at a given compression pressure. They discovered that binder concentration and compression pressure influenced disintegration time and that a decrease in binder concentration will necessitate increasing the compression pressure to produce satisfactory tablets.

The increase in relative density is a reflection of increased compression pressure, which has a great effect on disintegration time and dissolution. The increase in relative density reduces specific surface area, thereby reducing the rate of penetration of liquid into the core of tablets as a result of increase in the binding force between particles due to their close proximity. This reduces the pore space in which binder particles can swell when wetted before disruption of the tablet. ${ }^{44}$

The effect the concentration of binding agent has on disintegration could be due to the fact that for an immediate release tablet, the tablet needs to disintegrate into granules before deaggregating to fine particles. The deaggregation process involves the removal of the binder layer around the particles, and this is subject to the amount of binder coating the particles; thus, the higher the binder concentration, the slower the removal, since a higher concentration would lead to more binding. ${ }^{45}$ Changing the type of binder could have an effect on the disintegration time of tablets irrespective of compression pressure. Alebiowu and Itiola ${ }^{46}$ reported the effect of pregelatinized starch on disintegration time as being dependent on its concentration, but in the case of native starch, the effect on disintegration time was dependent on relative density (compression pressure). This was attributed to the fact that pregelatinized starch binders are cold water-swellable. ${ }^{47}$ This swellability would overwhelm the effects of compression pressure on the tablets. Concentration had a greater effect, since it leads to the formation of additional bonds. ${ }^{46}$ They also found that, with the combination of native/pregelatinized starch, the nature of the starch was completely different, having a stronger effect on dissolution than relative density as expected, because pregelatinized starches are cold water-swellable and have a higher swelling capacity, ${ }^{46}$ resulting in decreased dissolution times, unlike native starches in which lower swelling capacity was observed, which extended the dissolution time. The swellability of pregelatinized starches would undermine the effect that relative density would have on the penetration rate of water into the tablets. They concluded that the form or nature of a binder will have considerable influence on the effect that the relative density (compression pressure) of a tablet has on the disintegration and dissolution parameters.

\section{Conclusions}

Theoretically, an increase in compression force should lead to an increase in mechanical strength and a decrease in the release properties of tablets. In some practical situations, this trend may be distorted.

Tablet production is the result of a complex interaction between many factors involving the drug, excipient, the formulation, and processing variables. The effect is an interplay between variables, which are not easily understood close, because at times it does not follow a particular theoretical trend. The pharmaceutical formulator should try to understand and take advantage of these variables for the production of tablets with desired property. The degree and extent of interaction of these variables are not absolutely dependent on one factor. However, the uniqueness of the material characteristics of ingredients essentially determines the final properties of the finished tablet. It can, therefore, be concluded that the effect of compression pressure on tablets is complemented by factors such as the material properties of the drug and excipient, as well as the formulation and processing factors; all of these properties and factors, in turn, affect the mechanical strength and release properties.

\section{ORCID iDs}

Olutayo Ademola Adeleye (1) https://orcid.org/0000-0001-8716-4064

\section{References}

1. Kala S, Juyal D. Preformulation and characterization studies of aceclofenac active ingredient. Pharma Innov. 2016;5(9):110-119.

2. Patel S, Kaushal AY, Bansal AK. Compression physics in the formulation development of tablets. Crit Rev Ther Drug Carrier Syst. 2006;23(1):1-65.

3. Adetunji OA, Odeniyi MA, Itiola OA. Effect of formulation and process variables on the release, mechanical and mucoadhesive properties of ibuprofen tablet formulations. Acta Pol Pharm. 2015;72(2):357-365. 
4. Odeniyi MA, Ayorinde JO. Effects of modification and incorporation techniques on disintegrant properties of wheat (Triticum aestivum) starch in metronidazole tablet formulations. Polim Med. 2014;44(3):147-155.

5. Razavi SM, Gonzalez M, Cuitino AM. Quantification of lubrication and particle size distribution effects on tensile strength and stiffness of tablets. Powder Technol. 2018;336:360-374.

6. Adeleye OA, Femi-Oyewo MN, Odeniyi MA. Effect of compression pressure on mechanical and release properties of tramadol matrix tablets. Current Issues in Pharmacy and Medical Sciences. 2015;28(2):120-125.

7. Adeleye OA, Femi-Oyewo MN, Odeniyi MN. The effect of processing variables on the mechanical and release properties of tramadol matrix tablets incorporating Cissus populnea gum as controlled release excipient. Polim Med. 2014;44(4):209-220.

8. Joiris E, Di Martino P, Berneron C, Guyot-Hermann AM, Guyot JC. Compression behavior of orthorhombic paracetamol. Pharm Res. 1998;15(7):1122-1130.

9. Yadav P, Sahdev AK. Physics of tablet with compaction and com pression process for novel drug dosage form. Int J Adv Sci Res. 2018;3(4):28-34

10. Celik M. Compaction of multiparticulate oral dosage forms. In: Ghebre-Sellassie I, ed. Multiparticulate Oral Drug Delivery. New York, NY: Marcel Dekker; 1994:333-355.

11. Marshall K. Tablet punch forces. In: Lachman L, Lieberman HA Kanig JL, eds. The Theory and Practice of Industrial Pharmacy. $4^{\text {th }}$ ed. New Delhi, India: CBS Publishers; 2013:524-545.

12. Vodáčková $T$, Vraníková $B$, Svainová $P$, et al. Evaluation and comparison of three types of spray dried coprocessed excipient for direct compression. Biomed Res Int. 2018; article ID 2739428. doi:10.1155/2018/2739428

13. Shipar A, Wadhwa A, Varughese C. Affect of granule sizes, types and concentrations of lubricants and compression forces on tablet properties. Int J Pharm Sci Res. 2014;5(11):4893-4801.

14. Gamlen M, Dey D. Tablets under pressure. Innovation in Pharmaceutical Technology. 2012;42.

15. Heckel RW. Density-pressure relationship in powder compaction. Transactions of the Metallurgical Society of AIME. 1961;221:671-675.

16. Kawakita K, Lüdde KH. Some considerations on powder compression equations. Powder Technol. 1971;4(2):61-68.

17. Odeku, OA, Itiola OA. Evaluation of the effect of khaya gum on the mechanical and release properties of paracetamol tablets. Drug Dev Ind Pharm. 2003;29(3):311-320.

18. Adeleye OA, Odeniyi MA, Jaiyeoba KT. Evaluation of cissus gum as binder in a paracetamol tablet formulation. Farmacia. 2011;59(1):85-95.

19. Haresh TM, Bhumin $\mathrm{P}, \mathrm{Nehal}$ JS. Development of $\mathrm{pH}$-independent matrix type sustained release drug delivery system of propranolo hydrochloride. J Appl Pharm Sci. 2011;1(3):83-92.

20. Lachman L, Lieberman HA. Evaluation/quality control test. In: Khar RK Vyas SP, Ahmad JF, Jain KG, eds. The Theory and Practice of Industrial Pharmacy. $4^{\text {th }}$ ed. New Delhi, India: CBS Publishers; 2013:481-483.

21. Sun $\mathrm{CC}, \mathrm{Hao} \mathrm{H}$. Improving mechanical properties of caffeine and methyl gallate crystals by cocrystallization. Crysth Growth Des. 2008;8(5):1575-1579.

22. Nanjwade BK, Ali MS, Nanjwade VK, Manvi FV. Effect of compression pressure on dissolution and solid state characterization of cefuroxime axetil. J Anal Bioanal Tech. 2010;1(3):112. doi:10.4172/21559872.1000112

23. Shipar MA, Ashish W, Cherian V, Narinder K, Niranjani T. Effect of Compression Force on Tablet Hardness and Disintegration Time. Project B MT 13-3. Toronto, Canada: Toronto Institute of Pharmaceutical Technology; 2013-2014.

24. Ordu Jl, Abidde TO, Okafo SE. Evaluation of the binding properties of gum obtained from dried leaves of Cochoros olitorious on metronidazole tablets formulation. Pharma Innov. 2018;7(5):688-694.

25. Ordu Jl. Onyemelukwe Jl. Comparative assessment of ginger and cassava starch as a binder on ibuprofen tablet formulation. Int J Life Sci Res. 2018. doi:10.21276/ijlssr.2018.4.2.20

26. Alebiowu G, Itiola OA. The effects of starches on mechanical properties of paracetamol tablet formulations. I. Pregelatinization of starch binders. Acta Pharm. 2003;53(3):231-237.
27. Mistry AK, Nagda CD, Nagda DC, Dixit BC, Dixit RB. Formulation and in vitro evaluation of ofloxacin tablets using natural gums as binders. Sci Pharm. 2014;82(2):441-448.

28. Sharma A, Sharma S, Jha KK, Singh IS. Formulation, optimization and evaluation of mouth dissolving tablets of piroxicam using hydrotropic solubilization technique. Int Res J Pharm. 2017;8(12):91-98.

29. Wang J, Flanagan DR. General solution for diffusion controlled dissolution of spherical particles. J Pharm Sci. 1999;88(4):731-739.

30. Ibrahim MA, Dawes VH, Bangudu AB. The contributions of erosion, swelling, and porosity to theophylline release kinetics from cissus populnea polymer matrices. Drug Dev Ind Pharm. 2000;26(5):571-575.

31. El-Bagory I, Baraka N, Ibrahim MA, El-Enazi F. Formulation and in vitro evaluation of theophylline matrix tablets prepared by direct compression: Effect of polymer blends. Saudi Pharm J. 2012;20(3):229-238.

32. Santos JV, Batista $A E$, Pina $M$. The influence of the compression force on zidovudine release from matrix tablets. AAPS PharmSciTech. 2010;11(3):1442-1448.

33. Löbmann K, Flouda K, Qiu D, Tsolakou T, Wang W, Rades T. The influence of pressure on the intrinsic dissolution rate of amorphous indomethacin. Pharmaceutics. 2014;6(3):481-493.

34. Dedhiya MG, Woodruff CW, Menard FA, Rhodes CT. Relationship between compression profile and physical properties of lithium carbonate formulation. Drug Dev Ind Pharm. 1998;14(1):56-61.

35. Iranloye TA, Parrott EL. Effects of compression force, particle size, and lubricants on dissolution rate. J Pharm Sci. 1978;67(4):535-539.

36. Kalu VD, Odeniyi MA, Jaiyeoba KT. Matrix properties of a new plant gum in controlled drug delivery. Arch Pharm Res. 2005;30(7):884-889.

37. Wan SC, Heng WS, Wong LF. Relationship Between Swelling and Drug Release in a Hydrophilic Matrix, Drug Dev Ind Pharm. 1993;19(10):1201-1210.

38. Lin Z, Zhou D, Hoag S, Qiu Y. Influence of drug properties and formulation on in vitro drug release and biowaiver regulation of oral extended release dosage forms. AAPS J. 2016;18(2):333-345.

39. Marais AF, Song M, de Villiers MM. Effect of compression force, humidity, and disintegrant concentration on the disintegration and dissolution of directly compressed furosemide tablets using croscarmellose sodium as disintegrant. Trop J Pharm Res. 2003;2(1):125-135.

40. Audu-Peter JD, Ibrahim MA. Use of ternary diagram in formulation design of paracetamol tablets. Pharmaceutical and Biosciences Journal. 2016;4(3):10-16.

41. Onyishi I, Chime SA, Ugwu JC. Evaluation of binder and disintegrant properties of starch derived from Xanthosoma sagittifolium in metronidazole tablets. African Journal of Biotechnology. 2013;12(20):3064-3070.

42. Etzler FM. Particle adhesion in the pharmaceutical sciences. In: Gutowski, WC, Dodiuk H, eds. Recent Advances in Adhesion Science and Technology. New York, NY: CRC Press, Taylor and Francis Group; $2013: 125-143$.

43. Wang S, Peng X, Tang L, Cao C, Zeng L. Contact-hardening behavior of calcium silicate hydrate powders. Materials (Basel). 2018;11(12):2367. doi:10.3390/ma11122367

44. Nordström J, Persson A, Lazorova L, Frenning G, Alderborn G. The degree of compression of spherical granular solids controls the evolution of microstructure and bond probability during compaction. Inter J Pharm. 2013:442(1-2):3-12.

45. Pant S, Sharma PK, Malviya R. Evaluation of different concentration of binders on the dissolution profile of paracetamol tablets. Adv Biol Res. 2015;9(2):82-85.

46. Alebiowu G, Itiola OA. Compressional characteristics of native and pregelatinized forms of sorghum, plantain, and corn starches and the mechanical properties of their tablets. Drug Dev Ind Pharm. 2002;28(6):663-672.

47. Anwar $\mathrm{E}$, Khotimah $\mathrm{H}$, Yanuar A. An approach on pregelatinized cassava starch phosphate esters as hydrophilic polymer excipient for controlled release tablet. J Med Sci (Faisalbad, Pakistan). 2006;6(6):923-929. 